\title{
Evaluation of magnetic resonance imaging relaxation time in wrist cartilage with scapholunate ligament injury
}

\author{
I saac Chappell, Dannica L Sturgeon, Phil Lee, Terence E Mcl ff, E Bruce Toby, Kenneth J Fischer \\ University of Kansas, Kansas, USA
}

Correspondence: Kenneth J Fischer. Address: University of Kansas, USA. Email: fischer@ku.edu

Received: October 14, 2014

Accepted: June 1, 2015

Online Published: July 27, 2015

DOI : $10.5430 /$ ijdi.v2n2p96

URL: http://dx.doi.org/10.5430/ijdi.v2n2p96

\section{Abstract}

Objective: The overall goal of this research is to identify completely non-invasive in vivo markers of cartilage degeneration following wrist injury in order to facilitate assessment and treatment of wrist injuries and prevention of osteoarthritis as a result of injury. In this study, the transverse relaxation time, T2, from magnetic resonance imaging (MRI) of the wrist cartilage of subjects exhibiting unilateral scapholunate dissociation was analyzed to evaluate changes in the biochemical status of the cartilage in the wrist following injury.

Methods: Data collection consisted of MRI scans of the wrist using 2 separate 3T scanners. Fourteen subjects were analyzed, each subject completed scans to evaluate T2 relaxation times on both their injured and contralateral (normal) wrist. Scans were conducted with a maximum of $0.390625 \mathrm{~mm} /$ pixel in-plane pixel size and $1 \mathrm{~mm}$ slice thickness. A series of four time echo scans ranging from $15-80 \mathrm{~ms}$ were collected. T2 relaxation time for each subject was calculated by registering these echo time scans and fitting the corresponding intensity values to an exponential decay curve.

Results: The T2 results from all subjects indicated no statistically significant changes with presence of injury. The use of two separate MRI scanners of the same strength of magnet coil did not cause a significant change in measurement values.

Conclusions: Our data suggests that either T2 relaxation time does not change with the presence of scapholunate injury in the wrist or that the change was insufficient to be detected in this study. The results from this study may function as a baseline for future studies examining the potential positive effect surgical repair has on T2 relaxation times.

\section{Keywords}

Magnetic resonance imaging, T2 relaxation time, Scapholunate dissociation, Articular cartilage, Osteoarthritis

\section{I ntroduction}

Hand and wrist injuries commonly occur, and some can be debilitating. Scapholunate dissociation results from an injury to the scapholunate interosseous ligament (SLIL) and generally requires surgery to restore normal biomechanics and alleviate pain. The integrity of the SLIL is often compromised when a person injures their wrist ${ }^{[1]}$. A fall on the outstretched hand and motions involving rotation of the wrist are common causes of this type of injury ${ }^{[2]}$. If left untreated, scapholunate dissociation can lead to scapholunate advanced collapse (SLAC wrist) and associated osteoarthritis ${ }^{[3]}$. 
Normal wrist function cannot occur if stability is disrupted by injury to the SLIL. As the severity of the injury increases, the injury is more likely to progress to SLAC wrist ${ }^{[4]}$. Rupture of the primary stabilizing segment of the SLIL, the dorsal segment, nearly always leads to scapholunate dissociation and rotatory subluxation of the scaphoid ${ }^{[5]}$. The scaphoid rotates, and the lunate and scaphoid dissociate, resulting in abnormal stresses imposed on the articular surfaces ${ }^{[1]}$. The overall goal of this research is to evaluate non-invasive in vivo markers of cartilage degeneration following wrist injury in order to facilitate assessment and treatment of wrist injuries and prevention of osteoarthritis as a result of injury.

When diagnosing scapholunate dissociation, radiography is commonly employed. Standard posterior-anterior and lateral radiographs ${ }^{[1,2]}$ are often accompanied by additional radiographs with the wrist deviated in the radial and ulnar directions, as well as with the hand in a forceful grip. Direct non-invasive imaging of the ligaments, however, can only be achieved through magnetic resonance imaging (MRI) ${ }^{[1]}$.

The non-invasive, high contrast nature of MRI makes it an effective means of assessing cartilage, ligaments, and other soft tissues. MRI has been utilized frequently for imaging cartilage in osteoarthritis cases, and is emerging as a very useful modality for cartilage evaluation. Blumenkrantz et al. ${ }^{[6]}$ commented on the benefits of MRI for monitoring arthritis, stating that its multi-planar and high spatial resolution images provide excellent joint tissue visualization without the use of any ionizing radiation. The excellent contrast provided by MRI for soft tissues such as cartilage is cited by Gold et al. as making MRI the best assessment technique for injuries and repairs to cartilage ${ }^{[7]}$.

Many types of MRI techniques have been utilized for cartilage, including quantitative MRI for cartilage volume and thickness. To obtain cartilage volume and thickness information, images with high-resolution are needed to clearly identify the cartilage boundaries; fat-suppressed spoiled gradient echo (SPGR) and fast double echo and steady state (DESS) with water-excitation sequences are used to obtain these images. For biochemical cartilage evaluation, the most common MRI techniques are longitudinal relaxation time in the rotating frame (T1 $\rho)$, delayed gadolinium-enhanced MRI of cartilage (dGEMRIC), sodium MRI ${ }^{[7]}$, and transverse relaxation time (T2) ${ }^{[6]}$. T1 $\rho$ values are sensitive to cartilage hydration and the interaction of water with large macromolecules, and have thus been used to infer proteoglycan (PG) content. The dGEMRIC imaging sequence assesses the glycosaminoglycan (GAG) concentration in cartilage by measuring the amount of contrast agent uptake in the cartilage after a time period, via the associated T1 relaxation time ${ }^{[6]}$. Sodium MRI is sensitive to proteoglycan content in the cartilage because of the attraction of sodium to the fixed negative charge density of GAGs in the cartilage, the magnitude of which correlates to the PG content ${ }^{[8]}$.

T2-weighted MRI has been extensively used in the clinical setting to characterize alterations of tissue in injury or diseases because T2 is sensitive to the alterations of biophysical and biochemical environment of water molecules in tissue. Compared to other MRI techniques, T2 measurement in cartilage can be readily achievable in clinical setting without requiring additional hardware (as does sodium MRI), contrast agent administration (as for dGEMRIC), or highly specialized pulse sequences that are not commercially available (as for T1 $\rho$ ). Parametric T2 relaxation time provides added advantage in that the tissue contrast is, as a time constant, theoretically independent from confounding factors in T2-weighted images including proton density, longitudinal relaxation time, repetition time and radio-frequency probe inhomogeneity. It provides information regarding changes of hydration state and collagen fiber orientation of cartilage, which are markers for cartilage degeneration. When water molecules are immobilized in the cartilage by a healthy collagen-proteoglycan matrix, $\mathrm{T} 2$ of water in the cartilage is shorter due to the reduced molecular motion allowed by the intact matrix. With degeneration of the cartilage, water escapes entrapment by the macromolecules, and the T2 of the water becomes longer due to the increased mobility of water molecules. Thus, as cartilage degenerates and the collagen and proteoglycan is depleted, the T2 relaxation time of the cartilage increases as water becomes more mobile due to disruption of the matrix ${ }^{[6]}$.

The objective of this study was to evaluate changes in the biophysical and biochemical status of the cartilage in the wrist following scapholunate ligament injury by measuring the T2 relaxation times in the cartilage of human subjects. Our 
hypothesis was that the calculated T2 relaxation time would be higher in the cartilage of injured wrists than in those of contralateral (assumed normal) control wrists.

\section{Methods}

The study protocol was approved by the local Human Subjects Committee. Data was collected from MRI of the radiocarpal joints in fourteen human subjects who exhibited unilateral scapholunate dissociation (11 male, 3 female, ages 22-67, mean age 42.8). Prior to scanning, each subject's pain levels associated with completing a maximal grip strength task were recorded. Pain levels were measured on a 0-10 visual-analog scale (zero indicated no pain; ten indicated unbearable pain).

Data collection consisted of MRI scans of the wrist using (for 4 subjects) a Siemens Allegra 3T scanner or (for 10 subjects) a Siemens Skyra 3T scanner (Siemens Medical Solutions USA, Inc., Malvern, PA, USA). During the study, the Allegra scanner was decommissioned, and the Skyra scanner was used for subsequent subjects. The scan series included a set of 4 rapid succession fast spin echo scans with varying echo times (20, 40, 60, $80 \mathrm{~ms}$ for the Allegra scans and 15, 30, 46, $61 \mathrm{~ms}$ for the Skyra scans) to allow for the calculation of cartilage T2 relaxation time (see Figure 1). Each of the 4 scans lasted approximately four minutes. These scans were taken with the patient's wrist in a relaxed state. For the Allegra scans, the subjects would be positioned laying on their front or side with their arm outstretched into the bore (Superman position). For the Skyra scans, the subjects would lie on their back with their wrist by their side and their entire body would enter the scanner bore. Images collected by the Allegra were acquired at a frequency of $123.23 \mathrm{MHz}$ with a field of view of $100 \mathrm{~mm}$ $\times 100 \mathrm{~mm}(256 \times 256$ pixels at $0.390625 \mathrm{~mm} / \mathrm{pixel})$ and a slice thickness of $0.6(19$ slices $)$; TR was $3420 \mathrm{~ms}$, flip angle was 180 degrees, and NEX $=2$. Images collected by the Skyra were acquired at a frequency of $123.25 \mathrm{MHz}$ with a field of view of $90 \mathrm{~mm} \times 90 \mathrm{~mm}(320 \times 320$ pixels at $0.28125 \mathrm{~mm} /$ pixel $)$ and a slice thickness of $1 \mathrm{~mm}$ ( 20 slices $)$; TR was $3560 \mathrm{~ms}$, flip angle was 120 degrees, and NEX $=2$. For the Allegra scans, a custom surface wrist coil was used; while for the Skyra scans, a Siemens multi-channel volume hand/wrist coil was used. The imaging protocol and analysis were performed on both the injured and contralateral wrist, which had not experienced any known previous injuries. These scans were part of 4-hour subject sessions that included other wrist scans used for additional research studies.
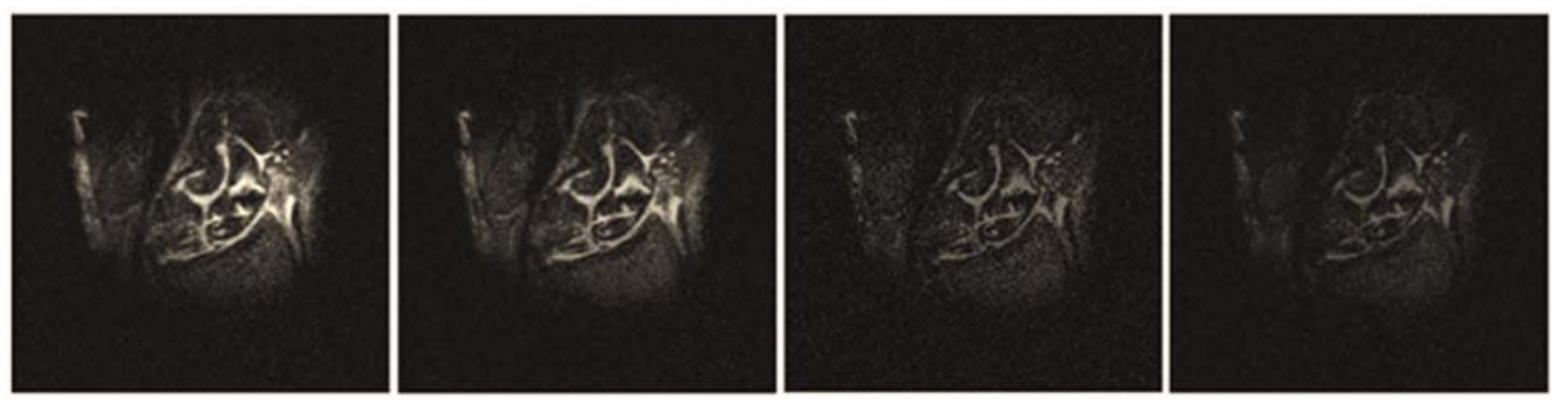

Figure 1. Example images from four echo times: From left to right, $20 \mathrm{~ms}, 40 \mathrm{~ms}, 60 \mathrm{~ms}$, and $80 \mathrm{~ms}$. The signal intensity can be seen to decrease as expected with increasing echo time

The four image sets obtained from the spin echo scans were first co-registered to minimize the effects of any position changes over the set of 4 scans. Very little motion was generally seen between time echo scans, with transformation matrices typically being near the identity matrix. Co-registration by normalized mutual information with linear interpolation (rigid body) was performed with Analyze 5.0 (AnalyzeDirect, Inc., Overland Park, KS, USA). The regions of radiocarpal and mid-carpal cartilage on the radius, lunate, scaphoid, and capitate bones in the wrist were then defined manually on the image set from the third echo time using Adobe Photoshop Elements 8.0 (San Jose, CA, USA). The same investigator defined all cartilage templates. Standard lab procedures include a second observer to provide quality control, 
and changes are only made by consensus. The image set from the third echo time was used to create this template because it was chosen as having the best overall visualization of individual cartilage surfaces. In addition, only the central portion of the cartilage was included in the template, to avoid partial volume effects at the cartilage edges (see Figure 2). Typically, the resolution of the time echo scans resulted in articular cartilage being only 3-4 pixels thick across many of the examined surfaces. This directly resulted in reproducibility of templates because only the central line of pixels was included in the template.
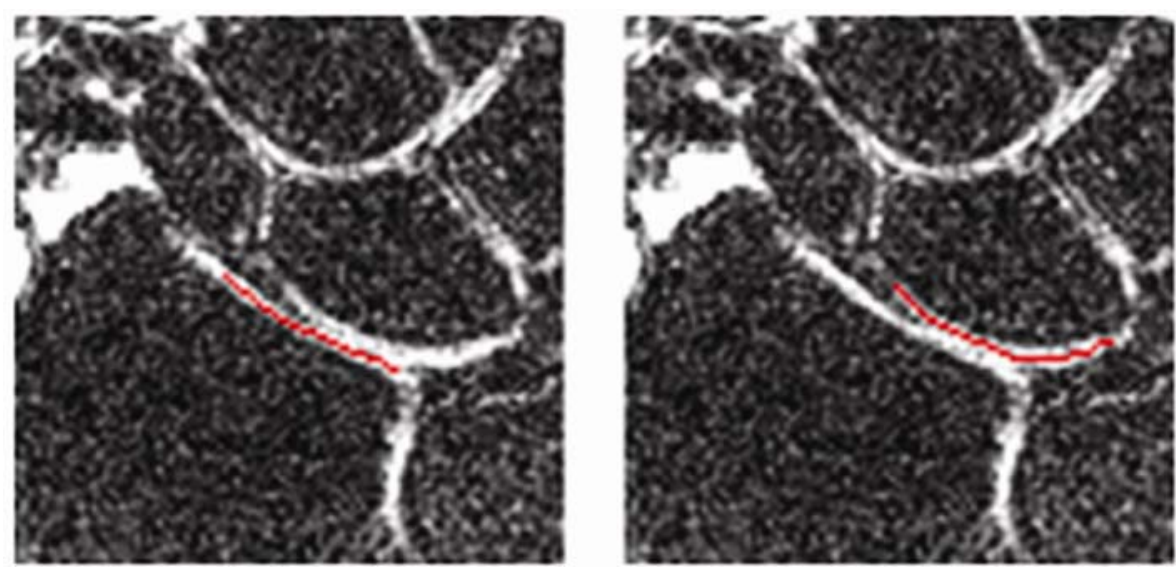

Figure 2. Examples of cartilage templates for radius (left) and Lunate (right) on the $60 \mathrm{~ms}$ echo time image

The data was then analyzed using a custom Matlab code to calculate the T2 value for each pixel of cartilage via linear regression to a semi-log plot of echo time vs. the natural logarithm of signal intensity. The signal intensity values were assumed to decay according to the following equation, dependent on the T2 time (T2):

$$
\mathrm{S}(\mathrm{TE}) \propto \mathrm{e}(-\mathrm{TE} / \mathrm{T} 2)
$$

where $\mathrm{S}$ is the signal acquired at different echo-times (TE) ${ }^{[6]}$. Pixels were deemed acceptable for statistical analysis after being filtered with a three-step process: 1) Individual pixel regressions that produced negative T2 times were discarded (these typically occurred near bone where image intensity was close to 0 across all time echoes); 2) pixels with a regression correlation coefficient (R2 value) of less than 0.6 were discarded; 3) pixels with outlier T2 times (outside the range of mean \pm 3 standard deviations), were discarded in an iterative fashion until all remaining pixels were within the acceptable range. This procedure reduced the possible spurious data due to spatial mismatch and over $60 \%$ of the original pixels defined as cartilage were accepted through the data filters.

Once the evaluation pixels were defined, the average intensity of the pixels was computed for each of the four echo times. A single T2 relaxation time was calculated from these four averaged values and recorded as the T2 value for the individual articular surface (While this method was considered most reliable, the difference between averaging $\mathrm{T} 2$ values of individual pixel locations was less than 1\%.). Descriptive statistics were calculated for the data and the mean T2 values for each cartilage surface were compared between the control and injured wrists, using two-way repeated measures ANOVA (with cartilage surface and injury state as factors) and a paired $t$-test on pooled (all cartilage surfaces) data $(p<0.05$ significant). In addition, regression analysis was performed to detect any relationships between the average T2 relaxation times, time from injury, and recorded pain levels in the study.

Because there were some concerns that the difference between Allegra and Skyra scanners may influence T2, we performed statistical analyses to test the influence of each scanner. Within the subject pool of this study, we had four subjects who had been scanned both on the Allegra and Skyra (at different time points, as both scanners were never active 
at the same time). For the comparisons, only uninjured wrists were used (firstly to limit any influence of injury and secondly, the T2 of the injured wrist is expected to vary over time while the uninjured should remain the same). First, a paired $t$-test was made on the data available for the four subjects to measure any direct influence the scanner had on the relaxation times. Second, a linear regression analysis was performed on the same data to verify whether the uninjured wrists remained similar over time. Finally, an unpaired $t$-test was performed on all available data between the two scanners.

\section{Results}

The results for the fourteen subjects showed no clear trends or patterns (see Table 1, Figure 3). No significant differences $(p<.05)$ were found. This finding does not support the study's hypothesis that injury would cause in increase in T2 relaxation time. Regression analysis showed that T2 values neither correlate with time from injury nor pain.

The R2 filter (the filter that removed the highest number of pixels) typically removed less than $5 \%$ of template pixels. The $\mathrm{R} 2$ values of all template pixels averaged around $0.85+/-0.10$ before the filter was applied. After the filter, the average R2 value increased by 0.02 and the standard deviation decreased by 0.01 .

The paired $t$-test for comparing the subjects across scanners was not significant $(p=.73)$. The unpaired $t$-test similarly showed no significant difference $(p=.84)$. In addition, the linear regression analysis was also not significant $(p=.81)$.

Although there were nosignificant differences, there is a possible trend noteworthy when plotting the data from acute to chronic illness states (see Figure 3). There appears there may be an initial increase immediately following the injury, as well as an eventual decrease to below uninjured values after 5 years of untreated injury.

As shown in Table 1, the values of T2 relaxation time do not have any clear outliers. The T2 times range between 30 and $88 \mathrm{~ms}$ with a mean value of $57.2+/-10.2 \mathrm{~ms}$ (57.4 +/- $10.8 \mathrm{~ms}$ for Uninjured; 57.0 +/- 9.7 for Injured).

Table 1. Individual T2 Times for Selected Cartilage Surfaces for All Subjects

\begin{tabular}{|c|c|c|c|c|c|c|c|c|c|c|c|c|c|c|c|c|c|c|c|}
\hline \multirow{2}{*}{ Sub \# } & \multirow{2}{*}{$\begin{array}{l}\text { Weeks } \\
\text { from } \\
\text { Injury }\end{array}$} & \multicolumn{2}{|c|}{ LR } & \multicolumn{2}{|l|}{ LC } & \multicolumn{2}{|l|}{ SR } & \multicolumn{2}{|c|}{ SC } & \multicolumn{2}{|c|}{ CL } & \multicolumn{2}{|c|}{ CS } & \multicolumn{2}{|c|}{ RL } & \multicolumn{2}{|c|}{ RS } & \multicolumn{2}{|c|}{ Average } \\
\hline & & $\mathbf{U}$ & $I$ & $\mathbf{U}$ & $I$ & $\mathbf{U}$ & $I$ & $\mathbf{U}$ & $I$ & $\mathbf{U}$ & $I$ & $\mathbf{U}$ & $I$ & $\mathbf{U}$ & $I$ & $\mathbf{U}$ & $I$ & $\mathbf{U}$ & $I$ \\
\hline 1 & 2 & 56 & 69 & 52 & 60 & 56 & 62 & 62 & 53 & 50 & 73 & 52 & 58 & 66 & 68 & 52 & 58 & 56 & 63 \\
\hline 2 & 2 & 30 & 51 & 38 & 47 & 36 & 45 & 61 & 45 & 38 & 42 & 52 & 51 & 41 & 51 & 36 & 42 & 41 & 47 \\
\hline 3 & 3 & 62 & 65 & 54 & 68 & 59 & 44 & 63 & 50 & 57 & 61 & 58 & 53 & 55 & 49 & 59 & 67 & 58 & 57 \\
\hline 4 & 24 & 64 & 55 & 47 & 55 & 66 & 68 & 66 & 67 & 59 & 52 & 53 & 67 & 52 & 51 & 66 & 69 & 59 & 60 \\
\hline 6 & 48 & 51 & 63 & 48 & 57 & 62 & 68 & 64 & 66 & 53 & 50 & 56 & 58 & 53 & 61 & 48 & 52 & 54 & 59 \\
\hline 8 & 100 & 60 & 59 & 50 & 63 & 67 & 73 & 52 & 88 & 52 & 48 & 61 & 61 & 70 & 76 & 50 & 73 & 58 & 68 \\
\hline 9 & 108 & 49 & 51 & 54 & 66 & 54 & 48 & 68 & 59 & 46 & 53 & 54 & 61 & 51 & 54 & 71 & 50 & 56 & 55 \\
\hline 10 & 283 & 62 & 65 & 52 & 44 & 55 & 71 & 60 & 69 & 49 & 55 & 54 & 53 & 54 & 58 & 49 & 59 & 54 & 59 \\
\hline 11 & 300 & 56 & 53 & 76 & 44 & 54 & 52 & 66 & 51 & 54 & 57 & 56 & 58 & 59 & 45 & 53 & 45 & 59 & 51 \\
\hline Ave & 672 & 56 & 56 & 52 & 54 & 61 & 60 & 66 & 61 & 56 & 53 & 59 & 58 & 54 & 56 & 56 & 59 & 57 & 57 \\
\hline
\end{tabular}

Note. Header Notation: First letter is bone of examined cartilage surface. Second letter is bone for articulating cartilage surface ( $\mathrm{L}=\mathrm{Lunate}, \mathrm{R}=\mathrm{Radius}, \mathrm{S}=\mathrm{Scaphoid}, \mathrm{C}=\mathrm{Capitate})$. Injury state is labeled as ' $\mathrm{U}$ ' for Uninjured (Normal) and 'I' for Injured. Ex: LR U is the cartilage surface of the Lunate that articulates with the Radius for the Uninjured/Normal wrist. All units in ms. 
Figure 3. T2 Ratio comparing injured and uninjured $\mathrm{T} 2$ relaxation times of all fourteen subjects, with increasing time since injury. The vertical lines indicate increasing time from injury. The ratio represents the value of the average injured $\mathrm{T} 2$ relaxation time over the average uninjured $\mathrm{T} 2$ relaxation time for the specific subject

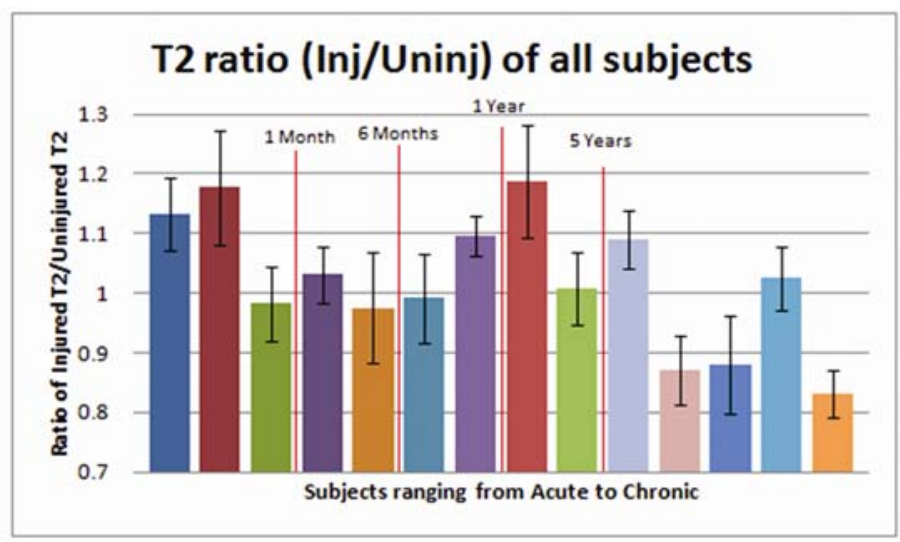

\section{Discussion}

Contrary to the initial hypothesis, the injured wrist cartilage of subjects exhibiting unilateral scapholunate dissociation did not produce higher T2 values than the contralateral, normal (control) wrist. No statistically significant differences were found. These findings suggest that either no changes have yet to occur; or that the magnitude of the changes are too small to detect, given the limitations produced by measuring biochemical changes in the relatively thin cartilage surfaces of the wrist.

Though the findings do not support the use of $\mathrm{T} 2$ relaxation time comparison for use in initial injury analysis, these measurements may be used as a baseline to determine if a change in $\mathrm{T} 2$ relaxation time does emerge after an injured wrists undergoes surgical repair. When observing the overall data (see Figure 3), we noticed a possible (but not statistically significant) pattern of higher T2 values in the injured state of acute subjects and decreased T2 values among subjects observed at more than 5 years from injury. This trend (though not statistically significant) suggests that after healing from the initial injury, long-term T2 values may decrease. Further study (data collection) is required to confirm this apparent phenomenon.

One encouraging finding of our analyses is that we found no significant difference between measuring $\mathrm{T} 2$ relaxation time using the two different scanners (Siemens 3.0 T Skyra and Siemens 3.0 T Allegra). This makes sense as T2 relaxation time should be a consistent mechanical property of the tissue that should not vary much between scanners with the same magnetic field strength. However, this result may not be generalizable to other scanners and/or wide variations in scanning protocol parameters. Also, the linear regression analysis (and lack of any significant relationship between $\mathrm{T} 2$ values on the two scanners) suggests that there was a substantial amount of random deviation occurring between scans of the same cartilage surface. Whether the variation was due to the different scan days or simply randomly measurement variation is unclear.

We used T2 relaxation time because of its ease of use and ubiquity across scanners. Mosher et al. ${ }^{[9]}$ reported that T2 values of normal cartilage range from $15 \mathrm{~ms}$ to $60 \mathrm{~ms}$, with variation in the $\mathrm{T} 2$ values spatially related to different locations in the cartilage, which is comparable to but generally lower than the values found in the current study. The higher values seen in our study may indicate influence from the synovial fluid and/or joint inflammation. Though we attempted to be careful to select a cartilage analysis template in the middle of the cartilage, there may still be some partial volume effects resulting in $\mathrm{T} 2$ value variation. Note that the wrist bones have very thin cartilage compared to larger joints analyzed in prior studies ${ }^{[9-10]}$.

Dunn et al. ${ }^{[10]}$ found significantly higher $\mathrm{T} 2$ values in knee cartilage of subjects who have arthritis when compared to healthy subjects in all but one of the knee compartments analyzed. In that study, healthy cartilage T2 values ranged from 32.1-35.0 ms, and osteoarthritic cartilage T2 values ranged from 34.4-41.0 ms. Although patients were divided into two 
OA groups in the study, mild and severe OA, no significant differences in $\mathrm{T} 2$ values were seen between the two groups. That suggests that $\mathrm{T} 2$ values may be able to delineate between healthy and degenerating cartilage, but once the cartilage degeneration process commences the $\mathrm{T} 2$ value may not significantly change during different stages of osteoarthritis.

Inflammation, which is associated with additional fluid surrounding the joint, due to the injury may be occurring in the wrist and may somewhat alter the results of the T2 time calculation. A study examining T2 values in the muscles of runners associated an increase in the value as a sign of inflammation/edema, however, they suggested there may be other possibilities as to the cause in the T2 value increases, such as the muscle exhibiting adaptations in its structure ${ }^{[11]}$.

This study had a number of limitations, including the relatively low number of subjects. The high amount of variability in the T2 times is another clear limitation, and it may be due to a number of factors. Motion artifact is always present to some degree in the images due to the nature of the human subject scans, especially with injury. Pixels may also experience some partial volume effects that could cause large changes in pixel intensity when exacerbated by motion. Error in the image registration may also lead to error in the mean $\mathrm{T} 2$ times, similar to motion artifact.

In the future, improved procedures could possibly aid in the reduction of motion related artifacts using multi-echo data acquisition per excitation. Scan time reduction and/or spatial resolution improvement could possibly be achieved with hardware improvements, such as increasing receiver coil channels. Additionally, given scanner and subject time constraints, if the hardware improved sufficiently additional time echo scans could be included and should result in more accurate $\mathrm{T} 2$ relaxation time measurements.

In conclusion, this study did not find a $\mathrm{T} 2$ relaxation time change with injury to the wrist.

\section{Acknowledgements}

Supported by the National Institute of Biomedical Imaging and Bioengineering of the National Institutes of Health under award R01EB008709.

\section{References}

[1] Ferreres A, Garcia-Elias M, Plaza R. Long-term results of lunocapitate arthrodesis with scaphoid excision for SLAC and SNAC wrists. J Hand Surg Eur Vol. 2009; 34: 603-8. PMid:19687078 http://dx.doi.org/10.1177/1753193409105683

[2] Atkinson LS, Baxley EG. Scapholunate dissociation. Am Fam Physician. 1994; 49: 1845-50. PMid:8203322

[3] Peterson B, Szabo RM. Carpal osteoarthrosis. Hand Clin. 2006; 22: 517-28. PMid:17097471 http://dx.doi.org/10.1016/j.hcl.2006.07.001

[4] Schmitt R, Froehner S, Coblenz G, et al. Carpal instability. Eur Radiol. 2006; 16: 2161-78. PMid:16508768 http://dx.doi.org/10.1007/s00330-006-0161-1

[5] Tanaka T, Ogino S, Yoshioka H. Ligamentous injuries of the wrist. Semin Musculoskelet Radiol. 2008; $12: 359-77$. PMid:19016398 http://dx.doi.org/10.1055/s-0028-1100642

[6] Blumenkrantz G, Majumdar S. Quantitative magnetic resonance imaging of articular cartilage in osteoarthritis. Eur Cell Mater. 2007; 13: 76-86. PMid:17506024

[7] Gold GE, Beaulieu CF. Future of MR imaging of articular cartilage. Semin Musculoskelet Radiol. 2001; 5: 313-27. PMid:11745048 http://dx.doi.org/10.1055/s-2001-19042

[8] Borthakur A, Shapiro EM, Akella SV, et al. Quantifying sodium in the human wrist in vivo by using MR imaging. Radiology. 2002; 224: 598-602. PMid:12147862 http://dx.doi.org/10.1148/radiol.2242011039

[9] Mosher TJ, Dardzinski BJ. Cartilage MRI T2 relaxation time mapping: overview and applications. Semin Musculoskelet Radiol. 2004; 8: 355-68. PMid:15643574 http://dx.doi.org/10.1055/s-2004-861764

[10] Dunn TC, Lu Y, Jin H, et al. T2 relaxation time of cartilage at MR imaging: comparison with severity of knee osteoarthritis. Radiology. 2004; 232: 592-8. PMid:15215540 http://dx.doi.org/10.1148/radiol.2322030976

[11] Marqueste T, Giannesini B, Fur YL, et al. Comparative MRI analysis of T2 changes associated with single and repeated bouts of downhill running leading to eccentric-induced muscle damage. J Appl Physiol. 2008; 105: 299-307. PMid:18450983 http://dx.doi.org/10.1152/japplphysiol.00738.2007 